\title{
Impact of impurity seeding and divertor conditions on transitions, pedestal structure and ELMs
}

\author{
M.G. Dunne \\ E-mail: mike.dunne@ipp.mpg.de
}

\begin{abstract}
Future devices will require a high scrape-off later (SOL) density and impurity seeding to avoid high-Z sputtering. However, these operational parameters are not included in present-day scaling laws, making extrapolations to larger devices difficult. As such, understanding the physics of such effects is vital in order to design the operational scenarios most favorable to high fusion gain. This review presents the favorable lowering of $\mathrm{L}-\mathrm{H}$ transition power by changing to metal walled devices and sumarises the effects currently thought to be responsible for how SOL geometry can play a role in determining this threshold. Experimental observations on changes to the pedestal structure with main ion fuelling and low-, medium-, and high-Z impurity seeding are presented. These results, from several devices, show that main ion fuelling or high density operation can result in a lower pedestal top pressure, and hence reduced stored energy, while impurity seeding can recover this lost pressure. Particular focus is given to nitrogen seeded discharges and the recovery of pedestal parameters (notably high $\mathrm{T}_{\mathrm{e}, \mathrm{ped}}$ ) in JET and AUG since the changeover to metal walls in these devices. Lithium seeding is also emerging as a strong actuator in pedestal dynamics, with results ranging from a prolonged inter-ELM period to completely ELM-free scenarios on different devices. ELM dynamics are also presented in each section, with nitrogen seeding offering a probe into the structure of the ELM and demonstrating the difference between the initial ELM crash, likely due to a sharp MHD event, and a prolonged second phase, the origin of which remains unkown. Finally, modelling of the pedestal in impurity seeded scenarios reveals a common effect in the position of the density profile. Either through mode excitation near to the separatrix or an altered fuelling profile, seeding of impurities results in an inward shift of the density profile. This inward shift improves MHD stability, allowing access to regions of higher pressure gradient and current density, and, hence, offering a promising operational scenario for future devices.
\end{abstract}


Impact of impurity seeding and divertor conditions on transitions, pedestal structure and ELMs2

\section{Introduction}

The impact of the scrape-off layer (SOL) conditions and impurities on the H-mode plasma has long been studied. Understanding these effects is of great importance for two main reasons. Firstly, deviations from confinement scaling laws, not only in the absolute values but also in trends have been observed in several machines, notably the density dependence in $\mathrm{H}_{98 \text {,y2 }}[1,2]$. New studies of the power degradation in specific scenarios also indicate that it may be less than quoted in the ITER-98 scaling[3], and also that it depends on the magnitude of the main ion gas puff[4]. Secondly, future devices have operational boundary conditions, such as a gas puff, installation of a metal wall, and a high radiated power fraction to reduce the power over the separatrix to keep high-Z sputtering to a minimum[5], conditions which are not easily contained in scaling laws as the impact they have on the plasma needs to be understood in detail to obtain a physics parameter. These two effects combined mean that the physics of such effects on the L-H transition, pedestal structure, and ELMs should be understood to make the extrapolations, even in a qualitative manner, to future devices.

This paper will summarise the recent work in understanding these effects from both experimental and theoretical points of view. The topics discussed in this paper are shown in a graphical overview in figure 1 and can be split into three main areas: unseeded scenarios, seeded scenarios, and the high-field side high density front (HFSHD). Unseeded scenarios relate to changes in the wall conditoning (also including changes to the wall materials) and main ion fuelling. Changing these parameters can have an effect on impurities in the plasma, as well as on the plasma density. The seeded scenarios are also split into two parts: first, divertor radiators, mainly used for divertor protection, such as nitrogen and neon; and secondly, core radiators, which are used to dissipate power before it reaches the SOL, such as argon, krypton, etc. The pedestal parameters in such seeded scenarios have been extensively studied in the metalwalled devices JET, AUG, and Alcator C-Mod, and impact the plasma by increasing the impurity content and the radiated power (either in the main plasma or the SOL). Finally, the HFSHD is a new feature being discussed. This is a region of high density which is poloidally localised to the high field side of the device, with a poloidal extent between the divertor entrance and the midplane (see figure 1 for an illustration). High density in this case means a density which is approximately one order of magnitude higher than the separatrix density[6]. This front is observed in JET and AUG when a gas puff is applied at sufficient heating power, and when the inner divertor reaches the fluctuating detachment state. The latest modelling with SOLPS[7] indicates that it is formed by drifts leading to an accumulation of neutral particles in the inner divertor which can then be ionised by power crossing the separatrix and reaching this region on the HFS. Once it has formed, the density in the HFSHD can be reduced When either the heating power is reduced, or a radiating impurity such as nitrogen is applied, i.e. when there is less energy available to ionise the neutrals in the region of the HFSHD. It should also be noted that the HFSHD is not a standard MARFE: it is localised 


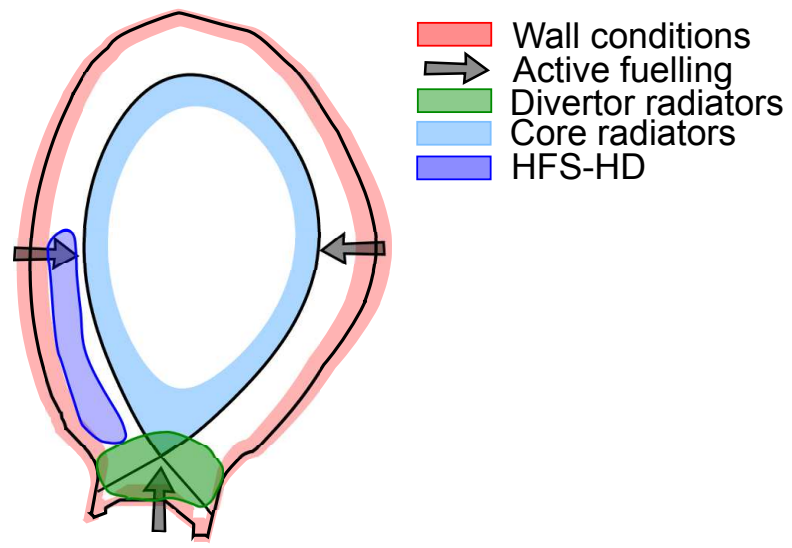

Figure 1. Overview of the topics discussed in this paper. Red indicates different wall materials and conditions, blue shows the radiation region for core radiators, black indicates an external gas puff, and green shows the region of SOL/divertor radiation.

below the midplane, its radiation is dominated by the electron radiation rather than impurity radiation and it is not an indication of a thermal instability; the HFSHD is routinely observed in many AUG discharges and, while it does not of itself cause an issue for stability or performance, the potential effect of such a front on the plasma will be discussed later in the paper.

Finally, ELM control will also have to be integrated into these radiative scenarios. While understanding the physics of ELM mitigation in present day experiments is an ongoing topic of research, using the fuelled and seeded experiments to understand ELM physics and make extrapolations is a valuable task. It is known that increasing the gas puff in a device increases the ELM frequency, for example, and this review will also look at the changes in the ELMs which occur with impurity seeding.

The rest of this paper is organised as follows: first, the impact of a different wall material and SOL geometry on the L-H transition power will be discussed. Following this, the observed effects of main ion fuelling and impurity seeding on pedestal structure and ELMs will be discussed. Finally, modelling of these scenarios will be presented and the observations of both the main ion fuelling and impurity seeding sections will be drawn together to form a unifying conclusion.

\section{Impact of impurities and divertor conditons on the L-H transition}

One of the strongest impacts of SOL conditions on H-mode access has been observed during the changeover from carbon to metal walls in AUG and JET. In AUG, the changeover was performed at a gradual rate, facilitating a detailed study of the effect of wall materials on the L-H transition power[8]. During this time, the L-H transition power was characterised during the so-called "Standard H-mode" pulse, which is performed as the first discharge on most days and exists on the high density branch of the LH power threshold. Figure 2 plots L-H transition power normalised to the ITPA scaling 
Impact of impurity seeding and divertor conditions on transitions, pedestal structure and ELMs4

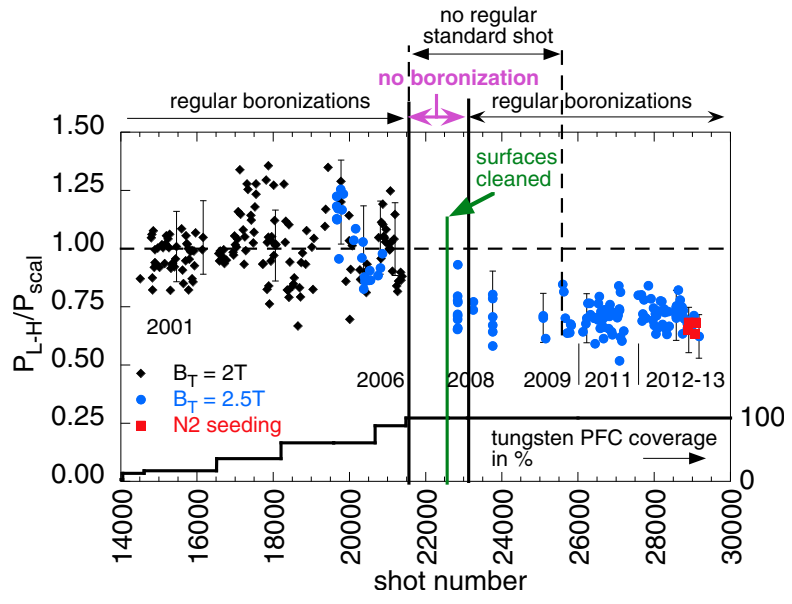

Figure 2. Power threshold normalised to the ITPA scaling law for a standard H-mode discharge in AUG over a period of 10 years around the installation of the metal wall. The blue points at $-2.5 \mathrm{~T}$ show a clear drop in the power required to access $\mathrm{H}$-mode after the surface cleaning. The red points also show a scan with nitrogen seeding, which indicates no $\mathrm{Z}_{\text {eff }}$ dependence on the $\mathrm{L}-\mathrm{H}$ power in these discharges. Figure reproduced with permission from $[8]$.

law[9] scaling showing that before the changeover occured the L-H transition power was well described by the scaling. Additionally, as the tungsten coverage was increased (blue points), the L-H transition power was also consistent with the scaling. Once the walls were cleaned (green line), the L-H transition power dropped by $20 \%$. A similar observation was made at JET, where the changeover from a carbon to a metal wall was completed in a single shutdown, with the L-H transition power decreasing by approximately 20\%[10]. Additionally, in the JET-C scans no density minimum could be found while it was recovered in the JET-ILW scans.

The dominant trigger for the LH transition at both devices is thought to be a critical edge radial electric field shear. As such, mechanisms which alter the edge radial electric field $\left(\mathrm{E}_{\mathrm{r}}\right)$ have been investigated. One possibility is that the lower $\mathrm{Z}_{\text {eff }}$ corresponds to lower dilution of the main ions, meaning they have a higher edge gradient and contribute to a higher Er. This has been tested in both AUG and JET by applying nitrogen seeding in the L-mode phase with contradicting results. In JET, an increase of the power threshold was observed[10], returning to powers seen in the carbon device, while on AUG[8] no change at all was noted (red points in figure 2). The latest comparison of edge density profiles at AUG[11] have shown a much steeper edge electron density gradient in the $\mathrm{W}$-wall machine, compared to a similar discharge in the $\mathrm{C}$-wall machine. This higher gradient then, again, would lead to a higher $\mathrm{E}_{\mathrm{r}}$ for a given level of heating power. This steeper gradient has been attributed to a higher reflection coefficient of main ions from the metal wall or the earlier onset of divertor detachment[11]. Further investigation of the origin of the $\mathrm{Z}_{\text {eff }}$ dependence in JET should also be undertaken to understand the effect and relate it to the AUG results.

Chaging the divertor geometry in L-H transition experiments has presented some 
challenges for the interpretation of the L-H power threshold and its scalings. Notably, several devices[12, 13, 14, 15, 16] have reported that the height of the x-point can alter the L-H threshold power by up to a factor of two. While a conclusive reason for these observations has not yet been found, recent JET experiments[17] focussing on smaller changes in the divertor geometry may help the interpretation of these experiments. In these experiments, the outer strikeline position was scanned from a horizontal target (HT) to a vertical target (VT). At the same plasma density, the L-H transition power was up to twice as high in the vertical target configuration as in the horizontal target, which is also observed at Alcator C-Mod in a similar scan[18]. Although differences in the $\mathrm{E}_{\mathrm{r}}$ well inside the main plasma were observed they were not considered strong enough to explain this large difference between the two configurations. Instead, the analysis focussed on the target $T_{e}$ profiles, which were different in the two cases due to different neutral recycling patterns. EDGE2D-EIRENE analysis of the two different geometries could match these profiles (strongly peaked near to the separatrix in the HT case and more flat in the VT case) and showed significantly different radial electric field profiles in the SOL. The HT E $\mathrm{E}_{\mathrm{r}}$ profile was strongly positive near to the separatrx while the VT profile was negative near to the separatrix and became slightly positive far into the SOL. These differences indicate that, with the same $\mathrm{E}_{\mathrm{r}}$ well inside the confined plasma, the shear in the outer part of the well can be different. The negative $\mathrm{E}_{\mathrm{r}}$ in the VT case indicates low shear, which, if this is the relevant parameter for the L-H transition, requires a deeper well in the plasma and hence more heating power.

While this has yet to be verified in the other cases, these experiments, and in particular the SOL analysis in the JET case, provide some insight into how the SOL conditions and geometry can influence the L-H transition. If veririfed, then the hypothesis of a critical $\mathrm{E}_{\mathrm{r}}$ shear in the outer part of the well as the trigger for the L-H transition would be given more support.

\section{Pedestal structure}

While the change from a carbon to a metal wall has positive impact by lowering the L-H transition power, it brings challenges to $\mathrm{H}$-mode operation in present day machines. Most notable among these is the requirement to avoid impurity accumulation, which, presently means operating with a gas puff or central wave heating. Gas puff operation is mainly aimed at increasing the ELM frequency which has two potential impacts: firstly, reducing the energy loss per ELM and hence reducing the sputtering of tungsten[19, 20] or increasing the tungsten flushing[21, 22]. As already mentioned, future devices will also be required to operate with divertor cooling to avoid excessive sputtering from the divertor tiles. It is of great interest to perform experiments with this combination of parameters in present day machines both the test our understanding of the physics of fuelling and seeding, and also to extrapolate this understanding to the design and operation of next generation devices. 

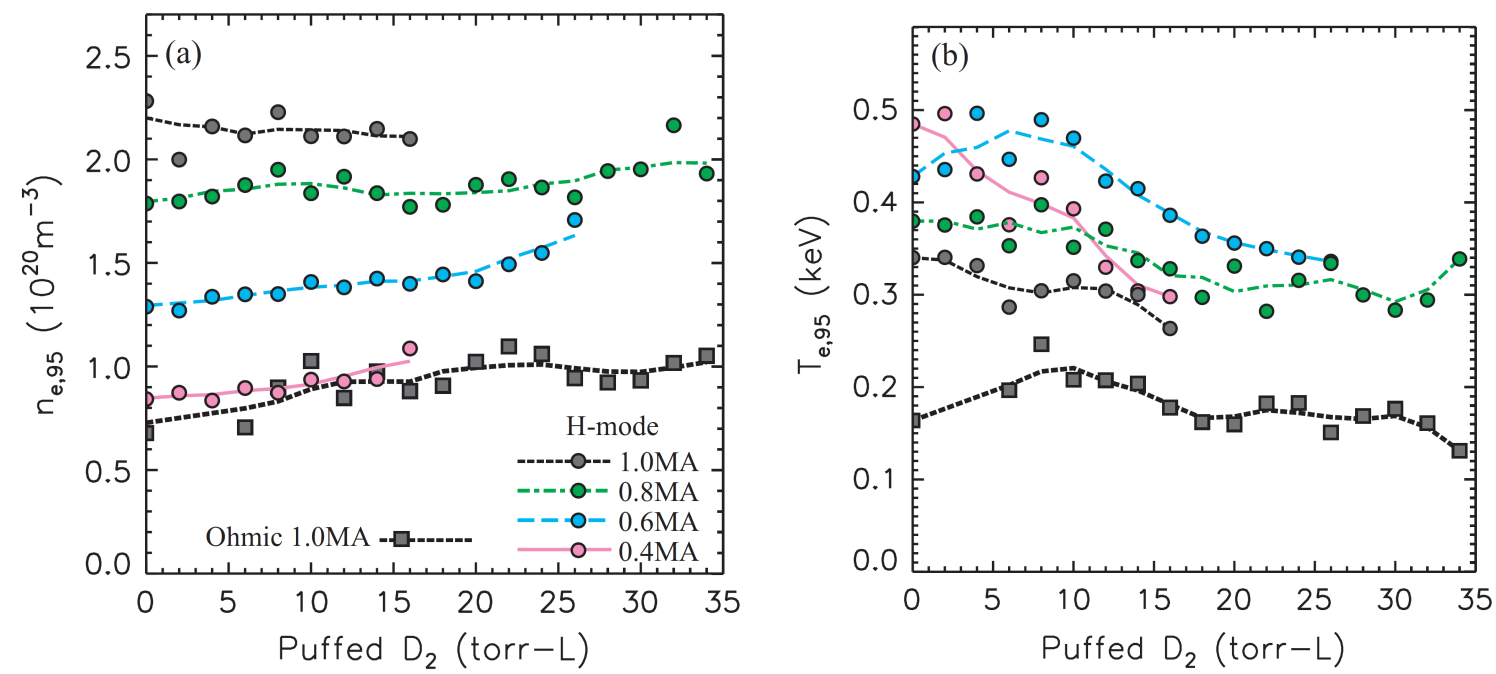

Figure 3. Pedestal top (a) density and (b) temperature as a function of D fuelling rate for a series of EDA H-modes in Alcator C-Mod. Different plasma currents are indicated by different colored symbols. The saturation of pedestal top density at $1 \mathrm{MA}$ is clear, unlike at the other currents. Despite this, the pedestal temperature decreases as it does at the other currents, leading to a pedestal pressure degradation. Figure reproduced with permission from [23].

\subsection{Main ion fuelling}

Confinement reduction via main ion fuelling is something which has been observed in many experiments on several tokamaks. Alcator C-Mod have reported a significant degradation of the pedestal in EDA H-mode with gas puffing[23]. In these experiments, a gas scan was performed at various plasma currents, as shown in figure 3. With increasing plasma current, the "natural", or recycling dominated, plasma density increased linearly, while the temperature at the pedestal top reduced as the total pressure increased less than linearly. At 0.4-0.8 MA, the pedestal top density (figure 3(a)) was observed to increase from these natural densities with increasing gas fuelling. However, at $1 \mathrm{MA}$ (grey circles), the density pedestal top value was fixed, independent of fuelling rate. In all cases, the temperature near the pedestal top (figure 3(b)) decreased with gas fuelling. At the lower plasma currents, this is at least partially offset by the increase of the pedestal top density, acting to conserve the pedestal top pressure. In contrast, a strong degradation of the pressure was observed at $1 \mathrm{MA}$, where the temperature decreased despite the constant density, resulting in an overall drop in both the pedestal top and global performance. The authors ascribed the difference in response of the density pedestal to a change in the particle confinement, which is highest in the $1 \mathrm{MA}$ plasmas and more L-mode like (lower gradients) at lower plasma currents.

Detailed analysis of the temperature and density profiles[24] showed an outward shift of the density profile relative to the temperature profile in the highly fuelled cases, with a large database showing that high global confinement could only be achieved at low values of the separatrix density normalised to global density. Although detailed 
stability analysis has not been done for these EDA H-mode plasmas, the effect here appears to be the opposite of the pedestal enhancement effects observed in DIII-D and NSTX (see section 3.4), where the density profile was shifted inwards and stability improved; this indicates that the density profile location can act to either enhance or degrade the pedestal.

Observations of increased pedestal density (or gas fuelling) on global and pedestal confinement on JET-C were also presented[25]. This analysis showed that the pressure at the pedestal top was inversely proportional to the density in the Type-I ELMy regime; once a threshold density or temperature was reached, the plasmas transitioned to a mixed Type-I/Type-II ELMy regime which allowed confinement comparable to the lowest density plasmas. This strong degradation of the pedestal with increased density has strong implications for operation of JET with the ITER-like wall, where the density is higher by default[2]; additional gas fuelling is also typically required, possibly making the situation worse[26]. In line with the C-Mod and JET-C results (in the Type-I ELMing regime only), the typical observation of the fuelled high triangularity baseline plasmas in JET-ILW is one of a higher density, lower pressure pedestal, leading to lower global confinement.

The effect of changing the wall material was also investigated on AUG[27], showing initially similar results. In a pair of discharges with no gas puffing, the pedestal density was higher and the temperature lower in the $\mathrm{W}$-wall device. Unlike the initial JET results, the effects cancelled each other out and resulted in a constant pedestal top pressure. This raises the important question of where this difference comes from. A hint could possibly come from the C-Mod observations, where severe pedestal degradation with density only occurred when the pedestal top density did not respond to gas puffing. This creates a picture of a pressure pedestal which, at low densities, does not vary with density; only the ratio of temperature to density changes. Once the pedestal density no longer responds to gas puffing, however, the pedestal top pressure starts to strongly degrade. Since the AUG pedestals are at low density with zero gas puff, the density pedestal will respond by increasing with an initial gas puff. In JET-ILW, some gas puff is usually required as the wall retention and recycling is too low to allow a sufficient fuelling of the core plasma[19], while the JET ones do not as they are already near their "natural" density.

\subsection{High-Z impurity injection}

While main ion fuelling is sufficient to reach detachment in present day devices, machines such as ITER will need to use impurity radiation in addition to neutral gas puffing in order to control high-Z sputtering from the divertor region[28]. Divertor temperatures below $15 \mathrm{eV}$ are required in order to keep sputtering to an acceptable level, which can only be achieved by medium- and high-Z impurities, such as nitrogen, neon, argon, etc. Gas puffing is also foreseen to be used to control the SOL density, so understanding how this combination of main ion fuelling and impurity seeding affects the plasma is 
important for scenario development. The most efficient use of an extrinsic radiator is seen with nitrogen, as it radiates strongly in the SOL and divertor regions, with little or no radiation in the main chamber plasma[28]. Neon, argon, and krypton have also been used to control the divertor heat loads in present day devices[29, 28].

The first method to reduce heat loads is increased density operation, typically via main ion fuelling. This approach has been compared with high density operation via argon puffing on the JT-60U tokamak[30]. This experiment demonstrates a decrease in confinement with increased density operation via main ion fuelling, as already shown for other devices. However, when argon puffing is used to achieve the same high densities, the thermal stored energy increases. This is due to the manner by which argon causes increased density operation; by increasing the density peaking. Taking two plasmas with comparable line integrated density, one with D fuelling and the other with Ar puffing, the Ar-puffed case shows a much more peaked profile. This means that, for the same line averaged density, the pedestal density is lower in the Ar case. For the same pedestal top pressure, this allows the temperature to increase, leading, via stiff profiles, also to a higher core temperature and hence thermal stored energy.

However, this form of high density operation comes at a price. Argon (also krypton and, to a lesser extent, neon) radiates inside the main plasma, causing the power flowing across the separatrix to reduce. Since the frequency of Type-I ELMs is correlated to $\mathrm{P}_{\text {sep }}$, the ELM frequency decreases. This has two negative effects: firstly, the ELMs become larger, impacting confinement and power loads, and, secondly, the ELMs are no longer effective at flushing high-Z impurities. While this is not an issue in carbonwalled devices, such as JT-60U, it is a serious problem for devices such as AUG, where a minimum ELM frequency must be maintained to avoid tungsten accumulation[21]. Recent modelling[31] done using a combination of ASTRA and STRAHL to determine the imapct of lowering the ELM frequency on the stability and performance of AUG plasmas showed that there is a minimum ELM frequency of $10 \mathrm{~Hz}$ required. Below this frequency, the model no longer converges, indicating a radiative collapse of the target plasma.

\subsection{Medium-Z impurities}

The alternative to using core radiators alone to achieve high density operation with low power to the divertor tiles is a combination of main ion puffing and medium-Z radiatiors, such as nitrogen or neon. A scan of the target power, i.e. the power density to plasma facing surfaces onto the divertor in Alcator C-Mod[32, 29] via four different methods was performed to compare all of the possibilities; main ion puffing only, argon seeding, neon seeding, and nitrogen seeding. As expected, in order to reach low target power with main ion fuelling, the confinement was decreased significantly. Since the aim of these experiments was to document the effects of power load reduction on performance near the L-H power threshold, argon seeding could also cause a back transition to L-mode via enhanced radation from within the confined plasma, thus significantly lowering the 
Impact of impurity seeding and divertor conditions on transitions, pedestal structure and ELMs9


Figure 4. Profiles of (a) temperature and (b) density for fuelled discharges in JETC (blue), JET-ILW (red), and JET-ILW with additional nitrogen seeding (black). The profiles in (c) and (d) are normalised to the pedestal top values of temperature and density, showing that the core gradient lengths have not increased and that the pedestal top value is determining the overall confinement change in JET-ILW. Figure reproduced with permission from [26].

confinement. Both neon and nitrogen, which radiated power predominantly in the SOL, allowed good confinement at low levels of target power, with nitrogen performing better than neon.

The changes in confinement with the different heat load reduction methods stemmed from a change in the pedestal properties. Increasing main ion fuelling and argon fuelling resulted in the pedestal density staying the same, but the temperature reducing. On the other hand, both the nitrogen and neon series existed at higher pedestal density and temperatures, with a small overall pressure pedestal change. This impact of medium$\mathrm{Z}$ impurities on the pedestal was exploited in recent JET-ILW experiments aimed at integrating a low divertor heat load with a high performance pedestal. As reported in the previous section, the higher density operation in JET-ILW compared with JET-C has led to lower confinement in high triangularity baseline plasmas[2, 26]. An example of how nitrogen seeding impacts the JET-ILW high triangularity baseline plasmas is shown in figure 4. Shown are (a) temperature and (b) density profiles from a fuelled JET-C discharge (blue), a similarly fuelled and heated JET-ILW discharge (red) and another similar discharge with additional nitrogen seeding (black). The figure, described in detail in Beurskens et al.[26] shows that both the pedestal top density and temperature are lower in the reference JET-ILW discharge than the JET-C counterpart. However, when nitrogen is added, the temperature pedestal recovers to the JET-C value while the density also recovers significantly. Figures (c) and (d) show the profiles normalised to the pedestal top values, indicating that the changes in the pedestal values propagate to the core via profile stiffness for $\mathrm{T}_{\mathrm{e}}$ and also via incresaed peaking at lower collisionality for $n_{e}$.

This lowering of global and pedestal confinement is most prominently seen when 
comparing low and high triangularity plasmas in the baseline configuration; increasing the triangularity no longer leads to higher performance as it did in JET-C[33]. The density is increased, as it was in JET-C and other devices, but the pressure remains the same, which is unfavourable for extrapolating this scenario. A plausible explanation for this lies in peeling-ballooning theory. The usual explanation for why high shaping increases pedestal confinement is that it alters the shape of the stability boundary in j-alpha space, allowing access to higher pressure gradients around the "nose" of the diagram, where the current driven peeling dominated region meets the pressure gradient driven ballooning dominated region. Linear MHD analysis offered a reliable interpretation of the JET-C data[34], and so was also used to analyse the JET-ILW data. The analysis in this case revealed two important findings. First, the pedestals appeared to be in the stable region of peeling-ballooning space, and, secondly, they were also closest to the high-n portion of the stability boundary, far from where plasma shaping impacts the boundary[35]. Once nitrogen seeding is applied to the low triangularity plasmas, a modest confinement increase of $\sim 15 \%$ is observed. However, in the high triangularity plasmas, the confinement can be increased by as much as $40 \%$, returning to levels seen in JET-C[33, 36].

A wider scan in heating powers has been made at AUG in combination with a seeding scan, focussing on two main chamber plasma shapes while keeping the divertor geometry the same[37]. A detailed power and seeding scan was performed at low triangularity showing that the highest attainable benefit of $\sim 40 \%[26,28]$ could only be obtained at the highest input power. The question remaining is if this is intrinsically a property of the plasma (i.e. high power/beta amplifying the effect which is seen at lower powers) or if it is an effect that only a small amount of nitrogen can be added at low power before the outer divertor detaches and confinement rolls over.

In contrast with the JET results, no change in the pedestal top density was observed with impurity seeding; the confinement improvement was driven by an increasing pedestal top temperature which propagates to the core plasma via stiff profiles[38]. While impurity seeding does not change the pedestal top density, increasing the triangularity of the plasma does, increasing the pedestal top density at constant temperature[39]. Contrary to the JET-ILW results, increasing the triangularity always increases the confinement in $\mathrm{AUG}-\mathrm{W}$, even at the lowest input power. Unlike confinement improvement via impurity seeding, higher triangularity increases the plasma density and thereby the confinement. When this is used in combination with impurity seeding, the result is a linear combination of the two effects; impurity seeding increases the temperature while triangularity increases the density, demonstrating a strong separation of the two effects.

The AUG results also offer some insight into the JET shaping experiments; the clear separation of temperature and density with shaping and seeding in this case indicates that there is no intrinsic amplifying effect of plasma shaping in combination with impurity seeding. It may be that impurity seeding in a low triangularity shape in JET-ILW moves the plasma into a region of peeling-ballooning space where confinement 
can be improved by increasing triangularity. Support for this hypothesis is given by a separate heating scan performed at JET[3]. Here, no difference between low and high triangularity is seen at low power. However, as the heating power is increased and the pedestal moves to a more favourable region towards the nose of the peeling-ballooning diagram at high $\left\langle j_{\|}>\right.$and $\alpha$, triangularity has a larger beneficial effect on the pedestal height. This interpretation is supported by modelling which also shows a larger effect of triangularity at higher global beta[4].

Problems with low ELM frequency when seeding high-Z gases have already been highlighted above. However, studies using impurity seeding in the metal-walled devices AUG and JET have helped to shed light on the structure of the ELM itself. An observation which was first commented on in AUG-W and JET-ILW was the "long" ELM, where, in contrast with the sharp crash usually reported in carbon devices, a prolonged phase of energy loss occurred[2, 27, 40]. Detailed analysis on both AUG[40] and JET[41] showed an ELM which consists of an initial short crash, similar to "typical" ELMs which was then followed by a second separate phase of energy loss. Further investigations and a comparison between similar discharges in AUG-C and AUG-ILW[27] showed that these longer ELMs did not just occur in metal-walled devices, but could also be accessed in carbon machines at similar pedestal parameters.

Analysis of a range of plasmas on both JET[41] and AUG[42] shows that the energy loss during the initial phase of the ELM (or, more accurately, during the short ELMs) scales inversely with collisionality, as already reported for a wide database over many carbon-walled devices[43]. In both machines, the energy loss in the second phase also scales with the pedestal top temperature, or any paramter related to this (such as collisionality or resistivity). ECE-imaging data show an increase in the number of ELM filaments for the long ELMs[42]. Zero filaments could be observed during the short ELMs with up to three filaments observed for the longest ELMs. This indicates an extended phase of energy transport from the main plasma to the SOL during the long ELMs, as expected. The AUG analysis also showed a correlation between the enery lost by the long ELMs and the divertor temperature[42], leaving two possible drivers for this behaviour: either there is a maximum pedestal top temperature/pressure or a minimum SOL/divertor temperature which allows the long ELMs to exist. Parameter scans in nonlinear ELM modelling for MAST[44] have shown that lowering the SOL temperature lowers the number of sets of filaments ejected by the ELM, lending support to the latter hypothesis. A further hypothesis has been put forward based on observations and modelling of recycling in JET-ILW $[45,19]$. The authors reported on a significant reduction in recycling after the ELM crash, commenting that this may be due to the complete outgassing of the clean tungsten targets due to ELM heat loads. The targets then act as a sink for particles for several milliseconds until the density profile in the plasma can start to recover. Further experiments will be necessary to determine whether the pedestal or the SOL/divertor determines the separation between long and short ELMs. 


\subsection{Low-Z impurity injection}

Medium- and high-Z impurity injection has been shown to improve performance and to change the nature of type-I ELMs. Experiments on NSTX, DIII-D, and EAST with low-Z impurities, notbaly lithium, have also shown improvements in the pedestal top pressure. This was first shown in a series of NSTX discharges[46] where the device was pre-conditioned with lithium and then lithium was actively added to the plasma during the discharge. The strong effect of the lithium conditioning process reduced the main chamber recycling so muhc that twice the main ion gas puff was requried to obtain the same line averaged density as in the reference case. In the process, the stored energy was also increased, mainly through an increased pedestal temperature and, hence, pressure. The drawback of this scenario is a rather high $\mathrm{Z}_{\text {eff }}$ and the fact that the boost to confinement was driven by with the formation of a classical ELMfree H-mode; i.e. impurity accumulation terminated the discharge. The authors of the NSTX study were, however, able to solve this issue with ELM triggering.

Detailed analysis of the pedestal profiles showed that the electron density profile was shifted inwards by approximately $2 \mathrm{~cm}[47]$. Linear peeling-ballooning calculations of the pedestals in reference and lithium seeded discharges show that the stability boundary is moved towards larger pressure gradients and current density as the pedestal pressure profile shifts inwards. In the cases presented, the shift was large enough that an ELM could not be triggered before a global MHD limit was reached, facilitating access to the ELM-free regime where the pedestal temperature could increase without being limited by edge modes.

A further set of experiments with lithium seeding in DIII-D[48] indicated confinement improvement but with a different driving mechanism. In this case, wall recycling was not thought to change significantly. Rather, the experiments were conducted in a regime which was susceptible to the occurrance of a bursty chirping mode (BCM), a transport instability which acted to flatten the density gradient in the middle of the pedestal. This mode was present in reference discharges, but was soon interupted by an ELM crash. When lithium was injected, the mode was sustained for much longer, further flattening the denstiy gradient and effectively shifting the density profile inwards. As in the NSTX experiments, the pedestal top pressure then increased due to increased access to higher pressure gradients in stability space, as shown in figure 5. Three experimental points are shown in this figure, two without lithium seeding or the BCM (green and blue) and one near the end of a $250 \mathrm{~ms}$ ELM free phase with lithium and the BCM (red), i.e. the beneficial scenario was not ELM-free, but the interELM time was extended significantly due to the movement of the stability boundary; the pedestal "recovery" time therefore took significantly longer until an ELM could be triggered, terminating the mode. The measured pressure gradients are shown in the inset, with the $\mathrm{Li}+\mathrm{BCM}$ pressure gradient shifted inwards away from the separatrix. Peeling-ballooning calculations show that the stability boundary in the BCM+Li case (red lines) is pushed further into the stable region than the reference cases (green and 




Figure 5. Pedestal stability in $\mathrm{j}-\alpha$ space for a set of low collisionality DIII-D discharges. Blue points show the measured values near the end of a naturally occurring $250 \mathrm{~ms}$ ELM-free period, green points show data near the end of a $20 \mathrm{~ms}$ ELM free period in an ELMing discharge, and red points show data from the end of a $250 \mathrm{~ms}$ ELM free period induced by the presence of the BCM and Li seeding. The lines show the calculated stability boundaries. The inset shows the measured pressure gradients from the respective discharges, with the $\mathrm{BCM}+\mathrm{Li}$ seeding pressure gradient shifted away from the separatrix, allowing access to higher pressure gradients, as indicated by the modelled stability boundary. Figure reproduced from [48].

blue lines), allowing the higher pressure gradient and current density to be reached.

Finally, a scenario was developed on EAST[49] which seems to be a combination of the above two scenarios. When lithium is injected into a discharge, an pre-existing edge coherent mode (ECM), thought to be a TEM, is further excited and results in a stable ELM-free scenario. In this case, confinement is not improved, but the discharge does not suffer from impurity accumulation, nor is confinement degraded. It is thought that the ECM provides sufficient outward transport to exhaust impurities. This raises the question of how well such a scenario wouold perform in a metal device, i.e. if the radial turbulent transport would be sufficient to exhaust high-Z impurities. Additionally, scenarios in which the ECM is already present would also need to be developed, as lithium injection itself is not enough to create such a mode. This may be an even more stringent criterion, as Alcator C-Mod regularly run in the so-called enhanced D- $\alpha$ (EDA) H-mode. EDA H-mode also features an edge coherent mode without high-Z impurity accumulation but has high collisionality access conditions which are not compatible with the high edge temperatures required in a reactor.

\section{Pedestal modelling}

Although we have touched on the interpretation of the experimental results presented in the previous sections, we now turn a more dedicated eye towards pedestal modelling. 
In order to understand how these changes take place, we first need a more general understanding of pedestal structure. The most successful model which predicts what pedestals will look like is the EPED model[50]. This posits that there are two constraints which will set the pedestal height and width, or, more accurately, its gradient. After an ELM crash, the model hypothesises that the pressure gradient will increase until it reaches a transport limit, which, based on many observations, appears to be a ballooning mode[51, 52]. Once this gradient limit is reached the pedestal then widens, growing further into the plasma until the combination of this critical gradient (and hence also current density) and pedestal width creates an unstable MHD mode, the peelingballooning mode[53], and an ELM occurs. In a simplification of the model, the gradient constraint can be translated into a constraint on the pedestal width, resulting in the formulation of the pedestal width as: $\Delta=\mathrm{C} \times\left(\beta_{\text {pol,ped }}\right)^{1 / 2}$. In order to understand how impurity seeding and main ion fuelling affect the pedestal, a predictive model where single parameters can be varied independently of each other is needed, and, thus, the assumptions of this model must first be validated.

One key issue with the EPED model is that it only considers the final pressure pedestal. It has been shown that the temperature and density pedestals recover after the ELM at different rates[54]; while this is irrelevant for a "steady-state" pre-ELM model, such dynamics should not be ignored when considering different fuelling profiles. This also impacts the choice of the transport limitation, which may then be different for temperature and density, rather than a pressure constraint. Detailed analysis of the pre-ELM JET-C and JET-ILW pedestals was performed with a view to testing the basic model assumptions[55]. This study presented some interesting findings. Firstly, that the model could sometimes predict the correct pedestal height, but the wrong width; i.e. the assumption on the width scaling can be incorrect. In particular, fuelling scans in JET-C and JET-ILW show different behaviours, as shown in figure 6. In JET-C (green points), main ion fuelling initially causes the pedestal to broaden at a constant gradient, leading to a higher pedestal. In JET-ILW (magenta points), in the same parameter range, fuelling also causes the pedestal to widen, but lowers the gradient, leading to an almost constant pedestal height. In contrast, a nitrogen seeding scan in JET-ILW (blue and red points) agrees well with the EPED assumption on the pedestal width scaling (dashed line). It should be pointed out here, that the fuelling scans are specific cases where the model has issues. In general, over a wide range of JET-C and JET-ILW plasmas[2], as well as scans on DIII-D, Alcator C-Mod, and AUG[50, 56], the EPED model predicts the pedestal height rather well, with $\sim 20 \%$ uncertainty, which is the combined uncertainty for both the model and measured data.

One significant issue is that the pedestal top density is an input for this model. However, changing the divertor geometry can change the density significantly. In one JET-ILW divertor geometry scan[33], the temperature and density pedestals react differently to impurity seeding. With an open pump throat, the density increases significantly with only a small increase in temperature. When the strike point is moved to a vertical target, the density now decreases when impurity seeding is applied, and 
Impact of impurity seeding and divertor conditions on transitions, pedestal structure and ELMs15

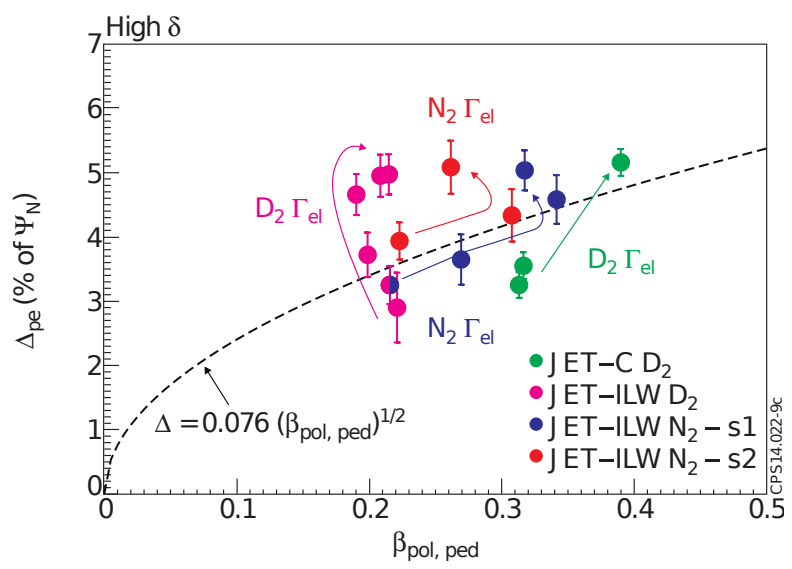

Figure 6. Pedestal width vs pedestal top poloidal beta for a JET-C fuelling scan (green), JET-ILW fuelling scan (magenta) and two nitrogen seeding scans (blue and red) in JET-ILW. The fuelling scans do not obey the scaling used in EPED-1.0 (dashed line), but the nitrogen scans are consistent with its assumptions. Figure reproduced from [55].

the temperature increases by a larger amount than in the HT configuration. These changes cannot easily be reconciled by transport processes thought to be dominant in the pedestal itself since only KBM transport is considered in the EPED model; rather, they are more likely to be due to changes in the SOL transport and the fuelling of the plasma. Simulations using EDGE2D-EIRENE have shown that these changes in divertor geometry could be responsible for an altered fuelling pattern, with neutrals more efficiently fuelling the plasma in the horizontal target (open pump throat) configuration[57].

Despite the drawbacks and shortcomings of the model, it is clear that both EPED and peeling-ballooning theory are useful frameworks within which at least trends can be described. At AUG in particular, much of the recent pedestal modelling has focussed on predictive pedestal modelling within the EPED framework[37]. Specific scans of global $\beta, \mathrm{Z}_{\mathrm{eff}}$, and plasma shaping were performed to compare the predictive capability of the model to the experiments described above. While the effect of beta and plasma shaping on the pedestal are well predicted by the model, the direct effect of $\mathrm{Z}_{\text {eff }}$ on the pedestal is negligible. At most, it generated a $\sim 5 \%$ increase of the pedestal top, which is far too low to describe the observed impact of nitrogen seeding, implying that another mechanism needs to be taken into account.

\subsection{Connecting SOL observations with pedestal modelling}

Further progress in interpreting the observations at C-Mod, JET, and AUG has been made by also taking SOL conditions into account as boundary conditions on the pedestal. Similarly to the NSTX[46, 47] and DIII-D[48] lithium experiments, it has been hypothesised that shifts of the pressure profile may be responsible for altering the pedestal stability. EDGE2D-EIRENE modelling at JET indicates that the separatrix 
temperature should decrease when nitrogen seeding is applied[35]. This was included into modelling of JET pedestals by assuming that the temperature profile (to which the density profile is also assumed to be locked) shifts radially inwards. The resulting stability diagrams show that access to higher pressure gradients is facilitated by such a shift. However, this requires that the density profile shifts with the temperature profile and also that a cooling of the separatrix would be reflected by an inward shift of the profile; this also implies a change of the profile gradient length inside the separatrix. Such a change due to impurity content cannot be ruled out, but a better understanding of pedestal structure is first required before this can be examined in more detail.

An inward shift is also hypothesised at AUG, but here focusses on only the density profile. This shift is motivated by the observation of the high field side high density (HFSHD) in the HFS-SOL of AUG and JET[58]. As mentioned in the introduction, the HFSHD is a region of high density localised at the HFS. It appears in discharges where a gas puff is applied and the inner divertor is in a partially or fully detached state. The power crossing the separatrix acts to ionise neutrals which accumulate in the HFS divertor region, creating this density front with a density approximately $10 \times$ higher than the separatrix density[6]. It has been associated with a fuelling inefficiency of the plasma; the core density does not rise significantly when the HFSHD is present, despite increasing the external gas puff. However, it could contribute to a diffusive or drift-driven fuelling of the plasma near to the separatrix[7], increasing the density in the outer edge of the pedestal. Since it has been already commented at C-Mod that pedestal density profiles are well described as self-similar[23], an increase at the separatrix is reflected by an effective outward shift of the density profile. When nitrogen seeding is applied, the power required to ionise the neutrals is reduced via SOL radiation, meaning that the density in the HFSHD is also reduced. This then decreases the separatrix density and the density profile shifts inwards, allowing higher pedestal top pressures to be reached. The resulting strong correlation between the reduction of the HFSHD and increase of $\mathrm{W}_{\mathrm{MHD}}$ is described by Potzel et al.[59] and shown in figure 7 for a database of nitrogen seeded discharges (black points) and also discharges with additional krypton seeding (red). The heating power, nitrogen puff rate, and plasma current were varied in this database, indicating that the effect is robust over a wide range of machine parameters.

Separate measurements of the location of the density profile for the power and nitrogen seeding scan, a subset of the discharges in figure 7, defined as the location in flux space of a particular density layer, have also shown such a correlation[60], implying a strong link between the HFSHD, the density profile location, and confinement. This radial shifting of the profile is the same effect observed to be triggered by the excitation of the BCM in DIII-D lithium seeded discharges[48], leading to increased pedestal stability, as shown in figure 5, and the reduced recycling in NSTX[47] leading to ELM-free Hmodes. In a test of this hypothesis, a density profile shift was included into the EPED framework at AUG[60]. This inward shift, at the maximum experimentally measured value of $0.01 \rho_{\text {poloidal }}$, showed an increase of the pedestal top pressure of $\sim 30 \%$. When combined with the small but positive impact of $\mathrm{Z}_{\text {eff }}$ via a reduction of the bootstrap 


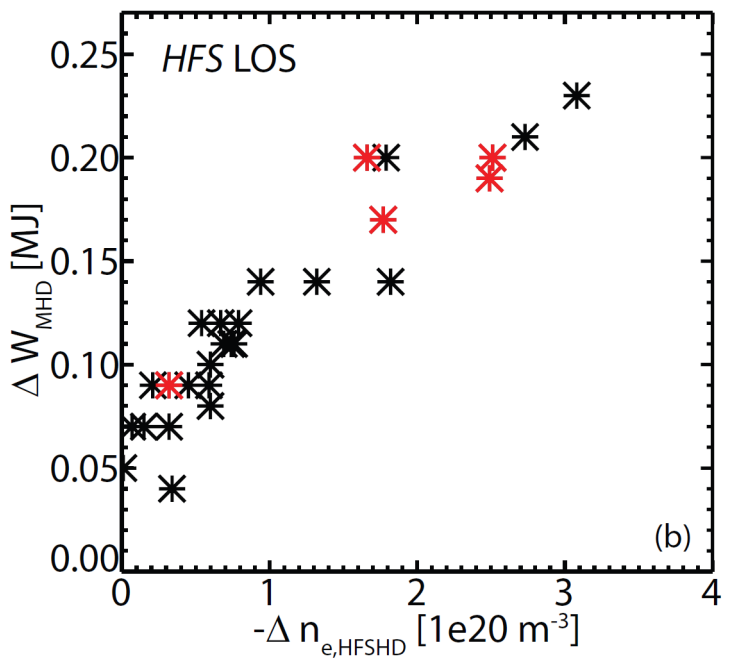

Figure 7. Change in plasma stored energy as a function of the reduction of the HFSHD. The black points are from nitrogen-only seeded discharges, while the red points have additional krypton seeding, indicating that the effect is independent of where or how the power is radiated; the power reaching the HFSHD region from the main plasma is the important factor here. Figure reproduced under the creative commons license from [59].

current, the results show a predicted increase of the pedestal top pressure of $\sim 35 \%$, which is consistent with the experimental observations.

The effect of the density profile location, modulated by the HFSHD, on pedestal stability would also explain the JET observations of improved confinement with nitrogen seeding, though this needs to be investigated in detail. It also explains the anticorrelation of pedestal and global confinement with separatrix density at C-Mod[24], where a strong outward profile shift was correlated with strongly degraded pedestals at a high main ion gas puff. Although not related to the formation of the HFSHD, the strong power degradation of JET-C high triangularity discharges[3] has also been ascribed to a source of neutrals at the upper divertor, implying that degrading plasma performance via the density profile shift can also have different sources. The conclusion from all of these observations, and also the lithium experiments from DIII-D, is that the pedestal, and, hence, global confinement are strongly dependent on the density profile location. Since the separatrix density is not strongly fixed by power balance requirements, it is free to vary according to the fuelling profile of the plasma. How the plasma is fuelled and how the pedestal density profile forms are thus becoming two of the most important questions which need to be answered when making predictions for future devices.

\section{Conclusions}

This review has detailed the changes to the L-H transition due to different wall materials and also by changes to the divertor geometry. While the effect of divertor geometry 
appears to have a promising explanation, the significant lowering of the L-H power threshold when moving to a metal wall has two possible causes. The first is a direct effect of $Z_{\text {eff }}$, possibly acting on turbulence growth rates. Evidence for this is seen in JET, but not in AUG, where a second effect, an altered density profile due to a higher wall-reflection coefficient for deuterium, is thought to be at play.

The main portion of this review has focussed on the pedestal height in the fully formed H-mode. Here, there are a wide variety of observations in different devices with different wall materials, impurity contents, and main ion fuelling rates. Beginning with fuelling, Alcator C-Mod showed degradation of the pedestal height with increasing gas puff when the density pedestal no longer responded to external fuelling. This is correlated with an outward shift of the density profile. Although this analysis of JET-C density profiles has not been done, a similar story is also present. Confinement degrades when the density pedestal top value does not change significantly with a gas puff; it may also be a reason for lower normalised confinement in high triangularity baseline JET-ILW discharges, as the density pedestal is naturally higher in metal machines.

AUG did not report any confinement loss in unpuffed discharges when moving from a carbon to a metal wall, despite similar phenomenology of a naturally higher density pedestal. While this may appear to be in contradiction to the JET results, it is likely that the plasma density can be efficiently increased by gas puffing in these low triangularity AUG plasmas, resulting in no net shift of the density gradient. It is also worth pointing out that most JET-ILW results to date show similar confinement to JET-C plasmas in low triangularity discharges, and also high triangularity hybrid (typically higher power) discharges. Again, this indicates that the fuelling efficiency may be an important parameter to consider when talking about main ion fuelling in connection with pedestal height and global performance.

A lowered fuelling efficiency is connected with the HFSHD; when this appears the density in the plasma is difficult to change. However, it could provide a source of particles via diffusion or drift-based transport due to the large density in the front. This fuelling mechanism would act to increase the density at the separatrix and shift the density profile outwards and hence reduce the pedestal top pressure, as observed in C-Mod. While this front has been observed on AUG and JET, detailed analysis connecting it to confinement has only been performed on AUG, showing a strong correlation between the density reduction in the HFSHD and confinement improvement. Predictive pedestal modelling has shown that the experimentally observed inward shift with nitrogen seeding, and not any changes in impurity content or global beta, explain the improvement in the pedestal top pressure. Since this front also reduces when the power in the SOL reduces, i.e. when input power is reduced or the power is radiated before it reaches the front, it is a plausbile mechanism for confinement improvement with nitrogen seeding. These experiments have typically been conducted in discharges featuring a gas puff an a partially detached inner divertor and, hence, the HFSHD.

A similar effect of impurities on the density profile had been reported earlier on NSTX and DIII-D, where lithium seeding acted to shift the density profile radially 


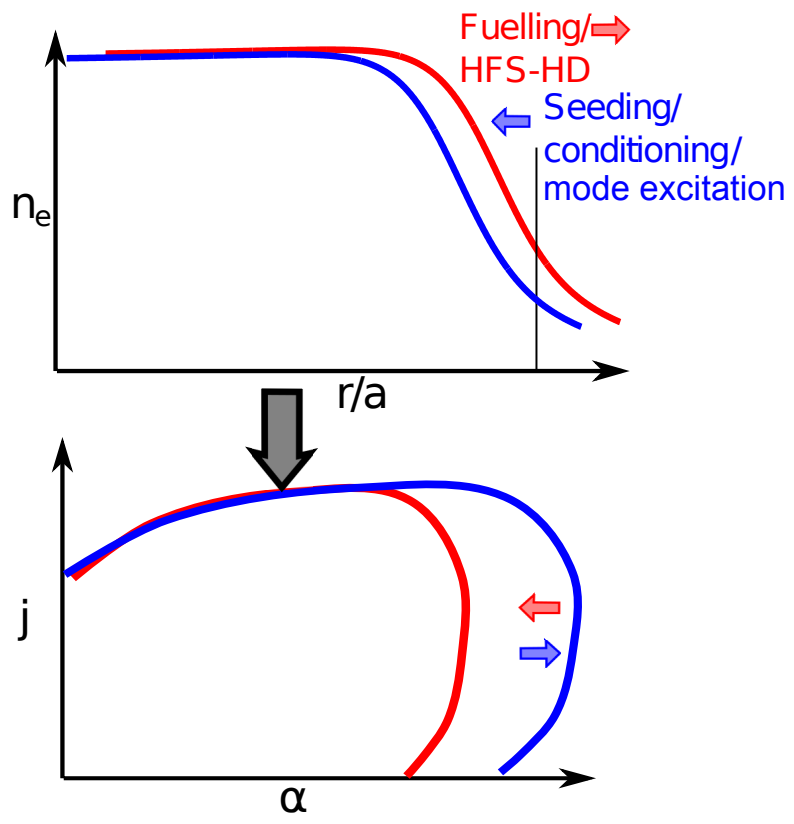

Figure 8. Top: sketch of mechanisms to shift the density profile inwards (blue) or outwards (red). Bottom: corresponding changes to the peeling-ballooning stability boundary; a pressure profile which lies further inside is more stable against peelingballooning modes.

inwards. In NSTX this was due to wall conditioning, while in DIII-D it was attributed to the excitation of a BCM in the middle of the pedestal which enhanced transport. In the NSTX case the effect was so pronounced that the peeling-ballooning stability boundary could no longer be reached, creating an ELM-free regime. Such a regime was also created with lithium seeding at EAST where an ECM provided sufficient radial transport to maintain a stable discharge.

All of these different experiments are linked by the density profile, its shape, and how it forms, as sketched in figure 8. Even with the same gradient, a density profile which forms closer to the separatrix will create a more unstable pressure pedestal, restricting access in j-alpha space and ultimately resulting in a lower pressure pedestal. If this can be corrected by, for example, seeding or mode excitation, access to higher pressure gradients can be facilitated and performance improved.

In the case of the profile shift in NSTX the ELMs completely disappear, but impurities also have different effects on the ELMs in ELMing H-modes. When impurities are seeded the nature of ELMs can also change. This ranges from a reduction of the frequency when radiation occurs inside the main plasma, to reducing the length of the ELM, typically observed with nitrogen seeding (and also fuelling) on both AUG and JET. How this latter effect works is still not clear; it could either be an effect of the pedestal top pressure or the temperature in the SOL. Further experiments with different impurities, as well as modelling advances, are required to isolate these effects from each other and determine the driving factor.

Experiments with impurities and main ion fuelling highlight how pedestal stability 
can be altered as a result of their effects on the density profile. This article has not touched on other aspects resulting from changes to the density profile, such as changes of radial transport. This may have further effects on the pedestal structure which also needs to be quantified and understood to project the findings from present day devices to future machines. These uncertainties and cause concern for a device such as ITER, which will couple gas fuelling and impurity seeding. Much work is needed to develop and validate our models of the SOL and how the density profile is formed, as well as for pedestal transport. Only then can predictions for performance in future devices be made with more certainty.

\section{Acknowledgements}

The author would like to thank M. Bernert, M. Beurskens, L. Casali, A. Diallo, L. Frassinetti, C. Giroud, J.W. Hughes, A. Järvinen, M. Leyland, A. Kallenbach, C. Maggi, R. Maingi, T. Osborne, S. Potzel, F. Reimold, M. Reinke, F. Ryter, S. Saarelma, P.A. Schneider, P. Snyder, H. Urano, E. Viezzer, J. Walk, M. Wischmeier, E. Wolfrum and the ITPA PEP group for fruitful discussions and valuable insights.

This work has been carried out within the framework of the EUROfusion Consortium and has received funding from the Euratom research and training

programme 2014-2018 under grant agreement No 633053. The views and opinions expressed herein do not necessarily reflect those of the European Commission.

\section{References}

[1] J. Schweinzer, A.C.C. Sips, G. Tardini, P.A. Schneider, R. Fischer, J.C. Fuchs, O. Gruber, J. Hobirk, A. Kallenbach, R.M. McDermott, R. Neu, T. Pütterich, S.K. Rathgeber, J. Stober, and J. Vicente. Confinement of improved H-modes' in the all-tungsten ASDEX Upgrade with nitrogen seeding. Nuclear Fusion, 51(11):113003, nov 2011.

[2] M.N.a. Beurskens, L. Frassinetti, C. Challis, C. Giroud, S. Saarelma, B. Alper, C. Angioni, P. Bilkova, C. Bourdelle, S. Brezinsek, P. Buratti, G. Calabro, T. Eich, J. Flanagan, E. Giovannozzi, M. Groth, J. Hobirk, E. Joffrin, M.J. Leyland, P. Lomas, E. de la Luna, M. Kempenaars, G. Maddison, C. Maggi, P. Mantica, M. Maslov, G. Matthews, M.-L. Mayoral, R. Neu, I. Nunes, T. Osborne, F. Rimini, R. Scannell, E.R. Solano, P.B. Snyder, I. Voitsekhovitch, and Peter De Vries. Global and pedestal confinement in JET with a Be/W metallic wall. Nuclear Fusion, 54(4):043001, apr 2014.

[3] C.D. Challis, J. Garcia, M. Beurskens, P. Buratti, E. Delabie, P. Drewelow, L. Frassinetti, C. Giroud, N. Hawkes, J. Hobirk, E. Joffrin, D. Keeling, D.B. King, C.F. Maggi, J. Mailloux, C. Marchetto, D. McDonald, I. Nunes, G. Pucella, S. Saarelma, and J. Simpson. Improved confinement in JET high $\beta$ plasmas with an ITER-like wall. Nuclear Fusion, 55(5):053031, may 2015.

[4] C.F. Maggi, S. Saarelma, F.J. Casson, C. Challis, E. de la Luna, L. Frassinetti, C. Giroud, E. Joffrin, J. Simpson, M. Beurskens, I. Chapman, J. Hobirk, M. Leyland, P. Lomas, C. Lowry, I. Nunes, F. Rimini, a.C.C. Sips, and H. Urano. Pedestal confinement and stability in JET-ILW ELMy H-modes. Nuclear Fusion, 55(11):113031, sep 2015.

[5] A. Kallenbach, M. Balden, R. Dux, T. Eich, C. Giroud, A. Huber, G.P. Maddison, M. Mayer, K. McCormick, R. Neu, T.W. Petrie, T. Pütterich, J. Rapp, M.L. Reinke, K. Schmid, 
Impact of impurity seeding and divertor conditions on transitions, pedestal structure and ELMs21

J. Schweinzer, and S. Wolfe. Plasma surface interactions in impurity seeded plasmas. Journal of Nuclear Materials, 415(1):S19-S26, aug 2011.

[6] S. Potzel, M. Wischmeier, M. Bernert, R. Dux, H.W. Müller, and a. Scarabosio. A new experimental classification of divertor detachment in ASDEX Upgrade. Nuclear Fusion, 54(1):013001, jan 2014.

[7] F. Reimold, M. Wischmeier, S. Potzel, L. Guimarais, D. Reiter, M. Bernert, and M.G. Dunne. The High-field Side High Density Region in SOLPS-Modeling of Nitrogen-seeded H-Modes in ASDEX Upgrade. Submitted to Journal of Nuclear Materials, 2016.

[8] F. Ryter, S.K. Rathgeber, L. Barrera Orte, M. Bernert, G.D. Conway, R. Fischer, T. Happel, B. Kurzan, R.M. McDermott, a. Scarabosio, W. Suttrop, E. Viezzer, M. Willensdorfer, and E. Wolfrum. Survey of the H-mode power threshold and transition physics studies in ASDEX Upgrade. Nuclear Fusion, 53(11):113003, nov 2013.

[9] Y R Martin, T Takizuka, and the Itpa Cdbm H-mode Threshold Data Group. Power requirement for accessing the H-mode in ITER. Journal of Physics: Conference Series, 123:012033, jul 2008.

[10] C.F. Maggi, E. Delabie, T.M. Biewer, M. Groth, N.C. Hawkes, M. Lehnen, E. de la Luna, K. McCormick, C. Reux, F. Rimini, E.R. Solano, Y. Andrew, C. Bourdelle, V. Bobkov, M. Brix, G. Calabro, a. Czarnecka, J. Flanagan, E. Lerche, S. Marsen, I. Nunes, D. Van Eester, and M.F. Stamp. LH power threshold studies in JET with Be/W and $\mathrm{C}$ wall. Nuclear Fusion, 54(2):023007, feb 2014.

[11] L M Shao, E Wolfrum, F Ryter, G Birkenmeier, F M Laggner, E Viezzer, R Fischer, M Willensdorfer, B Kurzan, and T Lunt. On the role of the edge density profile for the LH transition power threshold in ASDEX Upgrade. Plasma Physics and Controlled Fusion, 58(2):025004, feb 2016.

[12] Y Andrew, N C Hawkes, M G O'Mullane, R Sartori, M De Baar, I Coffey, K Guenther, I Jenkins, a Korotkov, P Lomas, G F Matthews, a Matilal, R Prentice, M Stamp, J Strachan, P De Vries, and Jet Efda Contributors. JET divertor geometry and plasma shape effects on the LH transition threshold. Plasma Physics and Controlled Fusion, 46(5A):A87-A93, may 2004.

[13] P. Gohil, T.E. Evans, M.E. Fenstermacher, J.R. Ferron, T.H. Osborne, J.M. Park, O. Schmitz, J.T. Scoville, and E.a. Unterberg. LH transition studies on DIII-D to determine H-mode access for operational scenarios in ITER. Nuclear Fusion, 51(10):103020, oct 2011.

[14] A Scaggion, Y Martin, and H Reimerdes. H-mode access with different X-point height in TCV. In 39th EPS conference on Plasma Physics, volume 1, Stockholm, 2012.

[15] Y. Martin, R. Behn, I. Furno, B. Labit, H. Reimerdes, and the Tcv Team. H-mode access during plasma current ramp-up in TCV. Nuclear Fusion, 54(11):114006, nov 2014.

[16] Y Ma, J W Hughes, a E Hubbard, B LaBombard, and J Terry. H-mode power threshold reduction in a slot-divertor configuration on the Alcator C-Mod tokamak. Plasma Physics and Controlled Fusion, 54(8):082002, 2012.

[17] E Delabie, A V Chankin, C F Maggi, H Meyer, T M Biewer, C Bourdelle, M Brix, I Carvalho, P Drewelow, C Guillemaut, N C Hawkes, J Hillesheim, D Keeling, A Meigs, L Meneses, F Rimini, G Sips, E Solano, M Stamp, J Svensson, Culham Science Centre, and Oak Ridge. The relation between divertor conditions and the L-H threshold on JET. In 42nd EPS Conference on Plasma Physics, Lisbon, 2015.

[18] Y. Ma, J. W. Hughes, A. E. Hubbard, B. LaBombard, R. M. Churchill, T. Golfinopolous, N. Tsujii, and E. S. Marmar. Scaling of H-mode threshold power and LH edge conditions with favourable ion grad-B drift in Alcator C-Mod tokamak. Nuclear Fusion, 52(2):023010, 2012.

[19] S. Brezinsek. Plasma-surface interaction in the Be/W environment: Conclusions drawn from the JET-ILW for ITER. Journal of Nuclear Materials, 463:11-21, 2015.

[20] G. J. Van Rooij, J. W. Coenen, L. Aho-Mantila, S. Brezinsek, M. Clever, R. Dux, M. Groth, K. Krieger, S. Marsen, G. F. Matthews, A. Meigs, R. Neu, S. Potzel, T. P??tterich, J. Rapp, and M. F. Stamp. Tungsten divertor erosion in all metal devices: Lessons from the ITER like wall of JET. Journal of Nuclear Materials, 438(SUPPL):S42-S47, 2013. 
Impact of impurity seeding and divertor conditions on transitions, pedestal structure and ELMs22

[21] A. Kallenbach, R. Dux, M. Mayer, R. Neu, T. Pütterich, V. Bobkov, J.C. Fuchs, T. Eich, L. Giannone, O. Gruber, A. Herrmann, L.D. Horton, C.F. Maggi, H. Meister, H.W. Müller, V. Rohde, A. Sips, A. Stäbler, and J. Stober. Non-boronized compared with boronized operation of ASDEX Upgrade with full-tungsten plasma facing components. Nuclear Fusion, 49(4):045007, apr 2009.

[22] R. Dux, V. Bobkov, A. Herrmann, A. Janzer, A. Kallenbach, R. Neu, M. Mayer, H. W. Müller, R. Pugno, T. Pütterich, V. Rohde, and A. C C Sips. Plasma-wall interaction and plasma behaviour in the non-boronised all tungsten ASDEX Upgrade. Journal of Nuclear Materials, 390-391(1):858-863, 2009.

[23] J.W Hughes, B LaBombard, J Terry, a Hubbard, and B Lipschultz. Edge profile stiffness and insensitivity of the density pedestal to neutral fuelling in Alcator C-Mod edge transport barriers. Nuclear Fusion, 47(8):1057-1063, aug 2007.

[24] J.W. Hughes, a. Loarte, M.L. Reinke, J.L. Terry, D. Brunner, M. Greenwald, a.E. Hubbard, B. LaBombard, B. Lipschultz, Y. Ma, S. Wolfe, and S.J. Wukitch. Power requirements for superior H-mode confinement on Alcator C-Mod: experiments in support of ITER. Nuclear Fusion, 51(8):083007, aug 2011.

[25] G. Saibene, R. Sartori, A. Loarte, D.J. Campbell, P.J. Lomas, V. Parail, K.D. Zastrow, Y. Andrew, S. Sharapov, A. Korotkov, M. Becoulet, G.T.A. Huysmans, H.R. Koslowski, R. Budny, G.D. Conway, J. Stober, W. Suttrop, A. Kallenbach, M. von Hellermann, and M.N.A. Beurskens. Improved performance of ELMy H-modes at high density by plasma shaping in JET. Plasma Physics and Controlled Fusion, 44:1769-1799, 2002.

[26] M.N.A. Beurskens, J. Schweinzer, C. Angioni, A. Burckhart, C.D. Challis, I. Chapman, R. Fischer, J. Flanagan, L. Frassinetti, C. Giroud, J. Hobirk, E. Joffrin, A. Kallenbach, M. Kempenaars, M. Leyland, P. Lomas, G. Maddison, M. Maslov, R. McDermott, R. Neu, I. Nunes, T. Osborne, F. Ryter, S. Saarelma, P.A. Schneider, P. Snyder, G. Tardini, E. Viezzer, and E. Wolfrum. The effect of a metal wall on confinement in JET and ASDEX Upgrade. Plasma Physics and Controlled Fusion, 55(12):124043, dec 2013.

[27] P A Schneider, L Barrera Orte, A Burckhart, M G Dunne, C Fuchs, A Gude, B Kurzan, W Suttrop, and E Wolfrum. Pedestal and edge localized mode characteristics with different first wall materials and nitrogen seeding in ASDEX Upgrade. Plasma Physics and Controlled Fusion, 57(1):014029, jan 2015.

[28] A. Kallenbach, M. Bernert, R. Dux, L. Casali, T. Eich, L. Giannone, A. Herrmann, R. McDermott, A. Mlynek, H.W. Müller, F. Reimold, J. Schweinzer, M. Sertoli, G. Tardini, W. Treutterer, E. Viezzer, R. Wenninger, and M. Wischmeier. Impurity seeding for tokamak power exhaust: from present devices via ITER to DEMO. Plasma Physics and Controlled Fusion, 55(12):124041, $\operatorname{dec} 2013$.

[29] M.L. Reinke, J.W. Hughes, a. Loarte, D. Brunner, I.H. Hutchinson, B. LaBombard, J. Payne, and J.L. Terry. Effect of N2, Ne and Ar seeding on Alcator C-Mod H-mode confinement. Journal of Nuclear Materials, 415(1):S340-S344, aug 2011.

[30] H. Urano, M. Nakata, N. Aiba, H. Kubo, M. Honda, N. Hayashi, M. Yoshida, and Y. Kamada. Roles of argon seeding in energy confinement and pedestal structure in JT-60U. Nuclear Fusion, 55(3):033010, mar 2015.

[31] L. Casali. Experimental studies and modelling of high radiation and high density plasma the ASDEX Upgrade tokamak. PhD thesis, LMU Munich, 2015.

[32] A. Loarte, J. W. Hughes, M. L. Reinke, J. L. Terry, B. LaBombard, D. Brunner, M. Greenwald, B. Lipschultz, Y. Ma, S. Wukitch, and S. Wolfe. High confinement/high radiated power H-mode experiments in Alcator C-Mod and consequences for International Thermonuclear Experimental Reactor (ITER) QDT = 10 operation. Physics of Plasmas, 18(5):056105, 2011.

[33] C Giroud, S Jachmich, P Jacquet, a Järvinen, E Lerche, F Rimini, L Aho-Mantila, N Aiba, I Balboa, P Belo, C Angioni, M Beurskens, S Brezinsek, F J Casson, I Coffey, G Cunningham, E Delabie, S Devaux, P Drewelow, L Frassinetti, a Figueiredo, a Huber, J Hillesheim, 
Impact of impurity seeding and divertor conditions on transitions, pedestal structure and ELMs23

L Garzotti, M Goniche, M Groth, Hyun-Tae Kim, M Leyland, P Lomas, G Maddison, S Marsen, G Matthews, a Meigs, S Menmuir, T Puetterich, G van Rooij, S Saarelma, M Stamp, H Urano, and a Webster. Progress at JET in integrating ITER-relevant core and edge plasmas within the constraints of an ITER-like wall. Plasma Physics and Controlled Fusion, 57(3):035004, mar 2015 .

[34] C. Giroud, G. Maddison, K. McCormick, M.N.a. Beurskens, S. Brezinsek, S. Devaux, T. Eich, L. Frassinetti, W. Fundamenski, M. Groth, a. Huber, S. Jachmich, a. Järvinen, a. Kallenbach, K. Krieger, D. Moulton, S. Saarelma, H. Thomsen, S. Wiesen, a. Alonso, B. Alper, G. Arnoux, P. Belo, a. Boboc, a. Brett, M. Brix, I. Coffey, E. de la Luna, D. Dodt, P. De Vries, R. Felton, E. Giovanozzi, J. Harling, D. Harting, N. Hawkes, J. Hobirk, I. Jenkins, E. Joffrin, M. Kempenaars, M. Lehnen, T. Loarer, P. Lomas, J. Mailloux, D. McDonald, a. Meigs, P. Morgan, I. Nunes, C. Perez van Thun, V. Riccardo, F. Rimini, a. Sirinnelli, M. Stamp, and I. Voitsekhovitch. Integration of a radiative divertor for heat load control into JET high triangularity ELMy H-mode plasmas. Nuclear Fusion, 52(6):063022, jun 2012.

[35] S. Saarelma, A. Järvinen, M. Beurskens, C. Challis, L. Frassinetti, C. Giroud, M. Groth, M. Leyland, C. Maggi, and J. Simpson. The effects of impurities and core pressure on pedestal stability in Joint European Torus (JET). Physics of Plasmas, 22(5):056115, may 2015.

[36] C. Giroud, G.P. Maddison, S. Jachmich, F. Rimini, M.N.a. Beurskens, I. Balboa, S. Brezinsek, R. Coelho, J.W. Coenen, L. Frassinetti, E. Joffrin, M. Oberkofler, M. Lehnen, Y. Liu, S. Marsen, K. McCormick, a. Meigs, R. Neu, B. Sieglin, G. van Rooij, G. Arnoux, P. Belo, M. Brix, M. Clever, I. Coffey, S. Devaux, D. Douai, T. Eich, J. Flanagan, S. Grünhagen, a. Huber, M. Kempenaars, U. Kruezi, K. Lawson, P. Lomas, C. Lowry, I. Nunes, a. Sirinnelli, a.C.C. Sips, M. Stamp, and S. Wiesen. Impact of nitrogen seeding on confinement and power load control of a high-triangularity JET ELMy H-mode plasma with a metal wall. Nuclear Fusion, 53(11):113025, nov 2013.

[37] M G Dunne, L Frassinetti, M Beurskens, M Cavedon, and R Fischer. Impact of nitrogen seeding on the AUG pedestal : experiments and modelling. In 42nd EPS Conference on Plasma Physics, Lisbon, 2015.

[38] G Tardini, R Fischer, F Jenko, a Kallenbach, R M McDermott, T Pütterich, S K Rathgeber, M Schneller, J Schweinzer, a C C Sips, D Told, and E Wolfrum. Core transport analysis of nitrogen seeded H-mode discharges in the ASDEX Upgrade. Plasma Physics and Controlled Fusion, 55(1):015010, jan 2013.

[39] M Beurskens, M G Dunne, L Frassinetti, M Cavedon, R Fischer, and F M Laggner. The role of carbon on the H-mode confinement in ASDEX Upgrade with a metal wall. Nuclear Fusion, 56:056014, 2016.

[40] P.A. Schneider, E. Wolfrum, M.G. Dunne, R. Dux, A. Gude, B. Kurzan, T. Pütterich, S.K. Rathgeber, J. Vicente, A. Weller, and R. Wenninger. Observation of different phases during an ELM crash with the help of nitrogen seeding. Plasma Physics and Controlled Fusion, 56(2):025011, feb 2014.

[41] L. Frassinetti, D. Dodt, M.N.a. Beurskens, a. Sirinelli, J.E. Boom, T. Eich, J. Flanagan, C. Giroud, M.S. Jachmich, M. Kempenaars, P. Lomas, G. Maddison, C. Maggi, R. Neu, I. Nunes, C. Perez von Thun, B. Sieglin, and M. Stamp. Effect of nitrogen seeding on the energy losses and on the time scales of the electron temperature and density collapse of type-I ELMs in JET with the ITER-like wall. Nuclear Fusion, 55(2):023007, feb 2015.

[42] L Frassinetti, M G Dunne, M Beurskens, E Wolfrum, A Bogomolov, and D Carralero. ELM behavior in ASDEX Upgrade with and without nitrogen seeding. Nuclear Fusion, Accepted f, 2016.

[43] A. Loarte, G. Saibene, R. Sartori, D. Campbell, M. Becoulet, L.D. Horton, T. Eich, A. Herrmann, G.F. Matthews, N. Asakura, A. Chankin, A. Leonard, G. Porter, G. Federici, G. Janeschitz, M. Shimada, and M. Sugihara. Characteristics of type I ELM energy and particle losses in existing devices and their Characteristics of type I ELM energy and particle. Plasma Physics 
Impact of impurity seeding and divertor conditions on transitions, pedestal structure and ELMs24

and Controlled Fusion, 45:1549-1569, 2003.

[44] S Pamela, G Huijsmans, A Kirk, I Chapman, and Mast Team. Effect of SOL Temperature on Filament Dynamics in MAST. In 41st EPS Conference on Plasma Physics, Berlin, 2014.

[45] D.M. Harting, S. Wiesen, M. Groth, S. Brezinsek, G. Corrigan, G. Arnoux, P. Boerner, S. Devaux, J. Flanagan, A. Järvinen, S. Marsen, and D. Reiter. Intra-ELM phase modelling of a JET ITERlike wall H-mode discharge with EDGE2D-EIRENE. Journal of Nuclear Materials, 463:493-497, 2015.

[46] M G Bell, H W Kugel, R Kaita, L E Zakharov, H Schneider, B P LeBlanc, D Mansfield, R E Bell, R Maingi, S Ding, S M Kaye, S F Paul, S P Gerhardt, J M Canik, J C Hosea, and G Taylor. Plasma response to lithium-coated plasma-facing components in the National Spherical Torus Experiment. Plasma Physics and Controlled Fusion, 51(12):124054, dec 2009.

[47] R. Maingi, T. H. Osborne, B. P. LeBlanc, R. E. Bell, J. Manickam, P. B. Snyder, J. E. Menard, D. K. Mansfield, H. W. Kugel, R. Kaita, S. P. Gerhardt, S. a. Sabbagh, and F. a. Kelly. EdgeLocalized-Mode Suppression through Density-Profile Modification with Lithium-Wall Coatings in the National Spherical Torus Experiment. Physical Review Letters, 103(7):075001, aug 2009.

[48] T.H. Osborne, G.L. Jackson, Z. Yan, R. Maingi, D.K. Mansfield, B.a. Grierson, C.P. Chrobak, a.G. McLean, S.L. Allen, D.J. Battaglia, a.R. Briesemeister, M.E. Fenstermacher, G.R. McKee, and P.B. Snyder. Enhanced H-mode pedestals with lithium injection in DIII-D. Nuclear Fusion, 55(6):063018, jun 2015.

[49] J.S. Hu, Z. Sun, H.Y. Guo, J.G. Li, B.N. Wan, H.Q. Wang, S.Y. Ding, G.S. Xu, Y.F. Liang, D.K. Mansfield, R. Maingi, X.L. Zou, L. Wang, J. Ren, G.Z. Zuo, L. Zhang, Y.M. Duan, T.H. Shi, and L.Q. Hu. New Steady-State Quiescent High-Confinement Plasma in an Experimental Advanced Superconducting Tokamak. Physical Review Letters, 114(5):055001, feb 2015.

[50] P.B. Snyder, R.J. Groebner, J.W. Hughes, T.H. Osborne, M. Beurskens, a.W. Leonard, H.R. Wilson, X.Q. Xu, Home Search, Collections Journals, About Contact, My Iopscience, and I P Address. A first-principles predictive model of the pedestal height and width: development, testing and ITER optimization with the EPED model. Nuclear Fusion, 51(10):103016, oct 2011.

[51] A. Diallo, J. W. Hughes, M. Greenwald, B. LaBombard, E. Davis, S-G. Baek, C. Theiler, P. Snyder, J. Canik, J. Walk, T. Golfinopoulos, J. Terry, M. Churchill, A. Hubbard, M. Porkolab, L. Delgado-Aparicio, M. L. Reinke, and A. White. Observation of Edge Instability Limiting the Pedestal Growth in Tokamak Plasmas. Physical Review Letters, 112(11):115001, mar 2014.

[52] A. Diallo, J.W. Hughes, S-G. Baek, B. LaBombard, J. Terry, I. Cziegler, A. Hubbard, E. Davis, J. Walk, L. Delgado-Aparicio, M.L. Reinke, C. Theiler, R.M. Churchill, E.M. Edlund, J. Canik, P. Snyder, M. Greenwald, and A. White. Quasi-coherent fluctuations limiting the pedestal growth on Alcator C-Mod: experiment and modelling. Nuclear Fusion, 55(5):053003, may 2015 .

[53] H.R. Wilson, S.C. Cowley, A. Kirk, and P.B. Snyder. Magneto-hydrodynamic stability of the H-mode transport barrier as a model for edge localized modes: an overview. Plasma Physics and Controlled Fusion, 48(5A):A71-A84, may 2006.

[54] A. Burckhart, E. Wolfrum, R. Fischer, K. Lackner, and H. Zohm. Inter-ELM behaviour of the electron density and temperature pedestal in ASDEX Upgrade. Plasma Physics and Controlled Fusion, 52(10):105010, oct 2010.

[55] M.J. Leyland, M.N.a. Beurskens, L. Frassinetti, C. Giroud, S. Saarelma, P.B. Snyder, J. Flanagan, S. Jachmich, M. Kempenaars, P. Lomas, G. Maddison, R. Neu, I. Nunes, and K.J. Gibson. The $\mathrm{H}$-mode pedestal structure and its role on confinement in JET with a carbon and metal wall. Nuclear Fusion, 55(1):013019, jan 2015.

[56] M. N. a. Beurskens, T. H. Osborne, P. a. Schneider, E. Wolfrum, L. Frassinetti, R. Groebner, P. Lomas, I. Nunes, S. Saarelma, R. Scannell, P. B. Snyder, D. Zarzoso, I. Balboa, B. Bray, M. Brix, J. Flanagan, C. Giroud, E. Giovannozzi, M. Kempenaars, a. Loarte, E. de la Luna, G. Maddison, C. F. Maggi, D. McDonald, R. Pasqualotto, G. Saibene, R. Sartori, E. Solano, 
M. Walsh, and L. Zabeo. H-mode pedestal scaling in DIII-D, ASDEX Upgrade, and JET. Physics of Plasmas, 18(5):056120, 2011.

[57] A Jarvinen. No Title. Plasma Physics and Controlled Fusion, accepted f, 2016.

[58] S. Potzel, M. Wischmeier, M. Bernert, R. Dux, F. Reimold, a. Scarabosio, S. Brezinsek, M. Clever, a. Huber, a. Meigs, and M. Stamp. Formation of the high density front in the inner far SOL at ASDEX Upgrade and JET. Journal of Nuclear Materials, pages 8-12, dec 2014.

[59] S Potzel, M Dunne, R Dux, L Guimarais, F Reimold, A Scarabosio, and M Wischmeier. On the high density in the HFS far SOL at ASDEX Upgrade and its impact on plasma confinement. In 42nd EPS Conference on Plasma Physics, Lisbon, 2015.

[60] Mike G Dunne. Predictive modelling of the impact of a radiative divertor on pedestal confinement on ASDEX Upgrade. In 57th Annual Meeting of the APS Division of Plasma physics, Savannah, GA, 2015. 